\title{
La traducción colectiva como proceso o producto. Reflexiones sobre el trabajo en colaboración a partir de casos de estudio concretos
}

\author{
Martha Celis-Mendoza \\ mcelis@colmex.mx \\ Universidad Iberoamericana, México
}

\begin{abstract}
Resumen
La traducción colectiva ha existido desde hace siglos, pero la reflexión sobre ella y su sistematización son mucho más recientes. La colaboración puede darse sobre el producto, con una autoría compartida, o sobre el proceso, donde intervengan dos o más agentes en tareas tan variadas como la gestión, la búsqueda terminológica o la revisión. Como ejemplo del primer caso se analiza una experiencia de colaboración en un proyecto editorial: la traducción al español de Reflexiones sobre traducción, de Susan Bassnett (2017), un trabajo conjunto de 39 colaboradores de diversas nacionalidades, edades y años de experiencia. Se contrasta con el trabajo cotidiano de un equipo de traducción, poniendo especial énfasis en cada una de las etapas del proceso y partiendo de casos concretos como el "Seminario de traducción permanente", de la UNAM (México), y el "Taller de traducción literaria", de la Universidad de la Laguna (España). Estos casos se ponen como telón de fondo para plantear la importancia de la enseñanza del proceso colaborativo de traducción, con base en la experiencia docente de varios colegas en programas de formación de traductores.
\end{abstract}

Palabras clave: traducción colectiva, formación de traductores, proceso traductivo, seminario de traducción, taller de traducción.

\section{Collaborative translation as process or product. Reflections on collaborative work based upon specific case studies}

\begin{abstract}
Collaborative translation has existed for centuries, but the reflection upon this issue and its systematization are much more recent. Collaboration can take place at product level, with a shared authorship, or at process level, translation work by two or more agents, who perform a number of tasks, such as management, terminology research, and/or revision. An experience of editorial translation in collaboration is presented as an example of the first case: the translation of Reflections on Translation, by Susan Bassnett (2017), into Spanish, which gathered together 39 collaborators of different nationalities, ages, and years of experience. This is contrasted with the everyday work of a translation team, paying special attention to task division as a process, taking as examples the case of unam's "Seminario de Traducción Permanente" (Mexico), and Universidad de la Laguna's "Taller de Traducción Literaria" (Spain). This is taken as a pretext to show the importance of instruction in the collaborative process of translation, based on the teaching experience of various colleagues in translator training programs.
\end{abstract}

Keywords: collaborative translation, translator training, translation process, translation seminar, translation workshop. 


\section{Traduction collaborative comme produit ou comme processus. Réflexions sur le travail collaboratif à partir d'études de cas spécifiques}

\section{Résumé}

La traduction collaborative existe depuis des siècles mais la réflexion sur ce sujet et sa systématisation sont beaucoup plus récentes. La collaboration peut se faire au niveau du " produit », avec une paternité d'œuvre partagée, ou au niveau du processus, au sein duquel interviennent au moins deux agents dans des tâches aussi variées que la gestion, la recherche terminologique ou la révision. Afin d'illustrer le premier cas, nous analysons une expérience de traduction éditoriale initiée en collaboration : la traduction en espagnol de Reflections on Translation, de Susan Bassnett (2017), travail conjoint de 39 collaborateurs de diverses nationalités, différents âges et années d'expérience. Nous le comparons au travail quotidien d'une équipe de traduction, en mettant en exergue tout particulièrement la séparation des étapes du processus ; nous prenons comme exemple le cas du "Seminario de Traducción Permanente" (UnAm, Mexique) et le "Taller de Traducción Literaria" (Universidad de la Laguna, Espagne). Ainsi, nous considérons l'importance de l'enseignement du processus collaboratif de traduction, sur la base de l'expérience pédagogique de plusieurs collègues au cours de leur formation en tant que traducteurs.

Mots-clés : traduction collaborative, formation de traducteurs, processus de traduction, séminaire de traduction, atelier de traduction. 


\section{Dos tipos distintos de colaboración: ¿proceso o producto?}

La práctica de la traducción en colaboración cuenta con referentes históricos, entre ellos la llamada Escuela de Traductores de Toledo, la cual ni se ubicaba exclusivamente en la que fuera la capital de Castilla en el siglo XII, ni era una "escuela" en el sentido moderno de la palabra, como nos lo recuerda Clara Foz en El traductor, la Iglesia y el rey (2000). Difícilmente pudieron haberse llevado a cabo trabajos como los emprendidos por incontables monjes a todo lo largo de Europa o los realizados al amparo de Alfonso x, El Sabio, si no hubieran existido comunidades de individuos dedicados a los diferentes aspectos de la labor de traducción, desde el cotejo de diferentes versiones del texto original, la investigación de equivalencias (lo que ahora llamaríamos búsqueda terminológica), hasta la transcripción y revisión de cada ejemplar de la obra traducida.

En el otro extremo de la historia nos encontramos con grupos de traductores, muchas veces anónimos y que además prestan sus servicios de manera voluntaria, para distintos proyectos de traducción globalizada, como pueden ser los Wiki-traductores o los Ted-translators, que ofrecen sus esfuerzos para que los materiales disponibles en esas plataformas puedan ser consultados por personas de distintas lenguas y culturas. Otro ejemplo importante de traducción colaborativa es el desarrollado por los más de 4.000 traductores que colaboran en Translators without Borders, asociación sin fines de lucro dedicada ofrecer sus servicios a organizaciones no gubernamentales en temas de salud, educación y ayuda humanitaria en situaciones de crisis. Todos estos grupos de trabajo parten de la convicción de que es fundamental superar las barreras de la comunicación para que la información pueda llegar al mayor número posible de personas, sin impor- tar cuál sea su lengua. El inglés, como antes sucediera con el francés y el latín, dejará en algún momento de ser la lingua franca. En su lugar, postula Umberto Eco, quedará la traducción (Eco, 1993). A este respecto, Carmen Alejandra González afirma de manera contundente: "La traducción es ubicua. Sí, la podemos encontrar en todo lugar y en constante movimiento" (2018, s/p). Su aseveración seguramente hará resonar en la mente de más de un lector la frase de Octavio Paz, "aprender a hablar es aprender a traducir", así como sus signos en rotación (Paz, 1971, p. 7).

Es precisamente a causa de ese constante movimiento que conceptos como traducción o traductor resultan a veces tan difíciles de definir y abarcar. Particularmente cambiante es por naturaleza el concepto de traducción colaborativa o traducción colectiva: "The field of collaborative translation understood as an enumeration of practices resists nominal definition: the field is non-essential, open and dynamic, and the position of any one collaborative translation event within its unique fabric of relations is constantly shifting" (Cordingley y Manning, 2018, p. 3). ${ }^{1}$ Los editores de Collaborative Translation atribuyen la reflexión más sistemática sobre el particular a la crisis en relación con la naturaleza del autor. Se ha cuestionado la autoría e incluso la existencia histórica de pilares fundamentales de la literatura occidental como Homero o Sha-

1 La discusión se extiende a la elección entre estos dos términos. Cordingley y Manning aclaran el matiz entre ambos, señalando el contraste entre el francés y el inglés: mientras que en francés el término en boga es traduction collaborative, por encima de traduction collective, que antes predominara, en inglés "the adjectival use of 'collective' in 'collective translation' might for some suggest a notion of mass translation factories or production lines, far from the idealized political harmony permitted in such friendly compounds as 'theatre collective' or 'artists' collective"' (Cordingley y Manning, 2018, p. 3). 
kespeare; del primero, por el carácter oral de los relatos primigenios, y del bardo inglés, por la participación de todo el engranaje que se pone en marcha en la puesta teatral, además de otras muchas hipótesis y consideraciones históricas y políticas. Partiendo de una serie de incertidumbres acerca de la importancia inamovible del autor como auctoritas suprema sobre el texto, y por lo tanto del traductor como coautor, Cordingley y Manning tratan de encontrar una respuesta analizando el problema desde distintos ángulos: "from dyadic interactions to networks of actors, modalities and technologies [...] given the overwhelming diversity of skills, knowledge and practices that emerge here, populating a vast landscape of translation, one may legitimately ask: what does 'collaborative translation' mean?" (Cordingley y Manning, 2018, p. 2).

Ante esta crisis sobre la figura del autor y del traductor resulta particularmente pertinente adoptar una postura como la que declara Selma Ancira, multipremiada traductora de poesía en griego y ruso, cuando afirma que sus editores valoran particularmente lo que reconocen como una actitud humilde. Ancira reconoce que la prosa "es un elemento en el que nado bien, pero no soy poeta y la poesía no es mi elemento. Entonces, recurro a alguien que en la poesía nada como pez en el agua" (Santoveña, Orensanz, Leal y Gordillo, 2010, p. 219). ${ }^{2}$ El reconocimiento de las propias capacidades, así como de las limitaciones, o como se les conoce actualmente en jerga pedagógica, las propias fortalezas y áreas de oportunidad, permiten

2 El testimonio de Selma Ancira aparece recopilado junto con los de treinta traductores literarios en Santoveña, Orensanz, Leal y Gordillo (2010). Por la naturaleza de esta obra, que recoge los comentarios de los traductores y los agrupa de manera temática, no de acuerdo con los colegas entrevistados, todas las futuras referencias a esta obra aparecen bajo el nombre de la editora principal. que ambas partes de la dupla de traductores que trabajan al alimón tengan la humildad de reconocer el valor de la aportación de cada una de ellas, en este caso el conocimiento más profundo de la lengua de partida y la capacidad de abstracción requerida para una buena prosificación por parte de uno de ellos y la capacidad de recreación poética de su contraparte.

Aunque la crisis de autor es algo relativamente nuevo, no lo son las colaboraciones, tanto en el ámbito de la creación poética (Sánchez Robayna, 2017), por ejemplo, como en el de la traducción:

From Antiquity to the Renaissance, translation was
frequently practised by groups comprised of special-
ists of different languages and with varied skills. At
the centre of translation teams, experts from various
cultures came together to find solutions to transla-
tion problems, and the acts of reading and rewriting
were often separated and multiplied between partici-
pants. (Cordingley y Manning, 2018, p. 1)

Destaca en el comentario de Cordingley y Manning la presencia de diversos agentes expertos provenientes de las diferentes culturas involucradas en el proceso, como ocurre por un lado en el ejemplo de las traducciones al alimón de Selma Ancira y Francisco Segovia, como por otra parte en los múltiples casos de traducciones especializadas en la actualidad, donde se requiere con frecuencia de la presencia de un experto en el tema, aunque no tenga conocimientos de traducción, para avalar la pertinencia terminológica del texto de llegada. Un ejemplo es la traducción de la obra de Oliver Sacks, El hombre que confundió a su mujer con un sombrero, obra de divulgación en el ramo de neurología y psiquiatría traducida por José Manuel Álvarez Flórez, y que incluyó la revisión científica de la traducción por el doctor Francesc Sabanés Magriñá, especialista que fuera vicepresidente de la Sociedad Catalana de Psiquiatría. 
Al hablar de traducción colectiva es importante distinguir entre el producto y el proceso. ${ }^{3}$ Una traducción al alimón, como la Novela de Giorgos Seféris, de Ancira y Segovia, puede considerarse un producto terminado que ostenta una autoría colectiva; lo mismo ocurre con Lineas conectadas. Nueva poesía de los Estados Unidos (2006), editado por April Lindner y su volumen gemelo, Connecting Lines, que incluye a cincuenta poetas mexicanos contemporáneos traducidos al inglés o estadounidenses traducidos al español por casi una decena de traductores a los que se da crédito desde la portada y en cada uno de los poemas traducidos. Es el caso de dos de las principales antologías de poesía traducida en México: El surco y la brasa, editada por Marco Antonio Montes de Oca (1974), y Traslaciones, por Tedi López Mills (2011). Ambas obras tienen la peculiaridad de contar entre sus páginas a poetas renombrados, quienes también incursionaron en el mundo de la traducción, como Alfonso Reyes, Octavio Paz, José Emilio Pacheco, David Huerta, Coral Bracho y Eduardo Langagne, entre muchos otros. La antología de López Mills destaca por la importancia que

3 Prefiero el adjetivo "colectiva" a "colaborativa" por el énfasis que intento dar a la figura del colectivo de traducción en sus distintas y muy fluidas variantes, así como por la presencia en numerosas publicaciones, particularmente en línea, de principios de esta década que identifican la traducción colaborativa con el fenómeno del crowdsourcing. O'Brien también lo identifica en lengua inglesa, precisamente a principios de esta década, de esta manera, aunque la diversidad de textos en la obra de Cordingley y Manning demuestra que el término no es restrictivo para ese tipo de colaboraciones: "The term has also come to be closely linked with the concepts of community translation, social translation, volunteer translation, fan translation, fansubbing and crowdsourcing. This close association of concepts is evident in the term ' $\mathrm{CT}^{3}$ ' (pronounced 'CT cubed'), which was coined by DePalma and Kelly (2008) to refer to 'Community', 'Collaborative technology' and 'Crowdsourcing" in the domain of localization’ (O’Brien, 2011). concede a los traductores, desde el subtítulo de la obra, Poetas traductores 1939-1959, hasta la disposición de los poemas seleccionados, agrupados no respecto al poeta autor del texto fuente, sino al poeta traductor, autor del texto meta.

Así entonces, cuando la traducción colectiva se refiere al producto, así sea un poema recreado por un poeta traductor, o un libro de divulgación de neurología como el texto de Oliver Sacks ya mencionado, se ha luchado, y en muchos casos se ha conseguido, dar crédito a los principales implicados en el proceso, es decir, los traductores que "firman" esa traducción o al menos a quienes se les reconoce explícitamente como colaboradores de la misma. ${ }^{4} \mathrm{No}$ es el caso, quizá en parte por la dificultad inherente del problema, de los distintos agentes involucrados en el proceso de la traducción, sea esta comercial, legal, especializada o incluso literaria. En los dos siguientes apartados de este texto intentaré analizar someramente ambas vertientes. En el apartado 2 presento como caso de estudio la traducción al español del libro de Susan Bassnett Reflections on Translation (2006). La obra reúne 39 de los ensayos publicados por la autora en dos revistas especializadas, ITI Bulletin y The Linguist. Gracias a la gestión de los editores de Bonilla Artigas se pudo llevar a cabo, con el apoyo del Fondo Nacional para la Cultura y las Artes en México (FONCA), este proyecto que tuve la oportunidad de coordinar desde sus inicios, contando con la colaboración de igual número de traductores, así como de otros agentes involucrados en el proceso de revisión, preparación de los distintos textos, etc. En el apartado 3 intento acercarme a la complejidad de la tra-

4 Sobre la cuestión de los derechos de autor para los traductores es sabido que los debates acalorados continúan a ambos lados del océano. Más complejo aún es el debate sobre los derechos de los colaboradores. 
ducción colectiva como proceso, tanto en los casos en que no se da crédito a los traductores, como puede ser la traducción comercial o especializada, como en los casos en que sí se reconoce la autoría de la traducción. Para este último aspecto refiero el caso de dos grupos que ejercen la traducción colectiva de manera similar, pero, como es natural, con sus características que los distinguen: el Seminario de Traducción Permanente de la Facultad de Filosofía y Letras de la Universidad Nacional Autónoma de México (UNAM) y el Taller de Traducción Literaria que desde hace más de veinte años dirige Andrés Sánchez Robayna en la Universidad de la Laguna, en Tenerife, España.

\section{La traducción colectiva como producto. Sobre la traducción de Reflexiones sobre traducción de Susan Bassnett}

Entre 2016 y 2017, 39 traductores nos embarcamos en la aventura de traducir una obra fundamental para los estudios de traducción: Reflections on Translation (2011), de Susan Bassnett. Esta es una obra muy particular que combina la erudición de su autora con un afán de compartir los temas que le apasionan tanto con profesionales de la traducción como con cualquiera que muestre interés en nuestra disciplina. En su reseña de la obra, Juan Carlos Calvillo afirma:

En Reflexiones sobre traducción Susan Bassnett se da a la tarea de recopilar los breves ensayos que aparecieron a lo largo de un período de diez años en el ITI Bulletin, la publicación periódica del Instituto de Traductores e Intérpretes del Reino Unido, y en The Linguist, la revista del Instituto de Lingüistas (CIOL). Con su estilo distintivo, su habitual ligereza y un sentido del humor que nunca se echa en falta, Bassnett le da rienda suelta en estos artículos informales a su más franca curiosidad en torno a la traducción, la identidad, el bilingüismo y la diglosia, la comunicación entre culturas y cualquier otro tema más o menos afín que la invite, como bien advierte el título, a reflexionar. (Calvillo, 2018, p. 312)

545

Lo que en un principio parecía ser un proyecto de traducción editorial más convencional, a cargo de una sola traductora, devino en una experiencia suigeneris. Por una parte, una serie de circunstancias logísticas no anticipadas en relación con la ayuda que recibimos por parte de PROTRAD (Programa de Apoyo a la Traducción del Fomento Nacional para la Cultura y las Artes de México) nos hizo darnos cuenta de la conveniencia de llevar a cabo la traducción de este libro por medio de un proyecto colaborativo. Por la otra, una serie de coincidencias afortunadas (en intereses, tiempos y voluntades) nos llevó a conformar el colectivo de traducción que ahora lleva por nombre Co-laboratorio Traductor. A él se han ido uniendo poco a poco algunos otros colegas que han colaborado con los miembros del colectivo original y que han aportado mucho a nuestras propias reflexiones, no solo sobre traducción, como señala el título de la obra de Bassnett, sino también sobre cuestiones lingüísticas en un espectro más amplio. En el equipo original contamos con traductores mexicanos y extranjeros; personas cuya lengua $\mathrm{A}, \mathrm{B}$ o $\mathrm{C}$ es el español, y cuyas lenguas maternas incluyen el español (mexicano, colombiano), el inglés, el ruso, el holandés, el búlgaro; así como jóvenes universitarios y otros jóvenes de espíritu; traductores especializados en literatura, en humanidades y en ciencias; traductores que con este trabajo teorizan sobre la traducción y profesores/investigadores que con esto regresan a la práctica de la traducción; desde peritos traductores, colegas especializados en subtitulaje y localización y una futura doctora en traducción, hasta traductores con mucha experiencia, pero cuya formación es empírica, solo con algunos cursos y mucha práctica, pasando por egresados de todas las etapas de 
formación académica:, licenciatura, diplomados y maestrías.

En los casos posibles, se seleccionó el tema del capítulo de acuerdo con el perfil del autor de la traducción. Uno de los dos capítulos dedicados a la traducción de noticias y el que aborda la cuestión de la nomenclatura de cuestiones geográficas se asignaron a dos colegas cuyas tesis de maestría están dedicadas a esos temas: las maestras Carmen Alejandra González Velázquez y Emma Sentíes Miranda, quien al respecto comenta:

Los artículos que asignó a los traductores coinciden con sus antecedentes profesionales, académicos, vocaciones e inclinaciones personales. Yo, por ejemplo, traduje un artículo sobre nombres geográficos, su traducción y las implicaciones políticas, culturales, comerciales de la misma. Es un texto breve con varios ejemplos, ameno, que puede leer tanto un especialista de la traducción como algún curioso del tema sin pretensiones académicas. Resulta que mi tema de tesis es éste mismo. Incluso algunos de los ejemplos que desarrollé en mi tesis se presentan en este artículo. [...] Mientras traducía me impactaron las coincidencias que había en afirmaciones y ejemplos. Era como si viera parte de mi tesis en este artículo. (Sentíes, 2018, s. p.) $)^{5}$

Otro de los colaboradores, César Octavio Martínez Benavidez, políglota con vasta experiencia en la docencia y la traducción de lenguas tan diversas como el francés, el catalán, el náhuatl, el esperanto y el quenya —una de las lenguas élficas creadas por J. R. R. Tolkien-, fue el encargado del primer capítulo del libro sobre cómo las distintas lenguas contribuyen a formar la identidad de los hablantes. El conmovedor artículo final, donde la autora hace

5 Calvillo abona al respecto en su reseña al señalar que "cada uno de ellos recibió la encomienda de traducir uno o dos capítulos del libro de acuerdo con su perfil, sus intereses o sus líneas de investigación" (Calvillo, 2018, p. 313). referencia a sus propias traducciones de la poesía de Machado tras la muerte de su esposo, fue preparado por un joven poeta, Rodrigo Círigo, ganador del premio de traducción Punto de partida por traducir "Little Gidding", uno de los Cuatro cuartetos de T. S. Eliot. E1 texto sobre las traducciones de las obras de Shakespeare en la actualidad lo ofrece el doctor Juan Carlos Calvillo, profesor especialista en la obra del dramaturgo inglés. El capítulo sobre la reivindicación de las traductoras mujeres lo entrega la doctora Socorro Soberón, traductora con vasta experiencia en el trasvase de textos sobre estudios de género y en traducción de literatura hecha por mujeres. Otro de los colaboradores, ávido lector de relato policial y enterado de las peculiaridades teóricas del tema por haber sufrido la tesis de maestría de su esposa, nos entrega su versión del artículo sobre la traducción de dicho subgénero. El capítulo sobre la traducción de la obra de poetas chinos a lenguas occidentales queda en manos de Radina Dimitrova, traductora de chino, quien ya antes de acercarse al artículo en cuestión estaba muy familiarizada con los poetas de esta lengua de los que habla Susan Bassnett; y, por último, qué mejor que dejar el texto más transgresor, que versa sobre la traducción de insultos, al colega más joven del equipo. Por mi parte, inmersa en ese momento en la investigación doctoral sobre Octavio $\mathrm{Paz}$, traductor de poetas de lengua inglesa, presento un capítulo sobre las dificultades de recrear el estilo en la traducción poética en un artículo donde se mencionan las opiniones de Ezra Pound, Charles Tomlinson y el propio $\mathrm{Paz}$, a quienes intenté analizar a profundidad en mi tesis.

La naturaleza del texto original, integrado, como ya se mencionó, por artículos que aparecieron en dos publicaciones especializadas distintas y en diferentes épocas, hace que sea más pertinente el trabajo colaborativo entre 
distintos traductores. Las delicias del diálogo entre colegas nos permitieron confirmar cómo el prójimo, el Otro, puede ver la misma cosa (en este caso una frase, un giro coloquial, etc.) desde otra mirada, con otra interpretación según el camino por donde lo haya llevado la investigación para dilucidar el significado del mismo "ladrillo" lingüístico. Así, los distintos capítulos de este libro, originalmente escritos como artículos, se constituyen como entes individuales, independientes entre sí, aunque unidos por un hilo conductor que es la reflexión sobre los vericuetos del traslado de una lengua a otra. Al respecto apunta Sentíes:

La heterogeneidad discursiva en este caso me parece de las aportaciones más valiosas que podemos encontrar en este libro. Susan Bassnett es una académica consolidada, con experiencias de vida muy particulares y sobre todo diversas, que ha entrado en contacto íntimamente con varias culturas, pues ha vivido en distintos países en alguna etapa de su vida. Todo esto significa que hay tanta diversidad en el discurso como numerosos son los artículos que ha escrito y los que aquí se traducen (2018, s. p.).

Mercedes Guhl, reconocida traductora, profesora y formadora de traductores, realizó sus estudios de posgrado bajo la guía de Susan Bassnett. En ocasión de la presentación del libro en las instalaciones de la Librería Bonilla, comentó que en la versión española "lograron que, a pesar de que son muchos los traductores, el estilo y la voz de Susan se perciban como un hilo común a todos los artículos del libro" (Guhl, 2018, s. p.). ${ }^{6}$ En la reseña que hizo de la obra, Calvillo apunta que "contrario a lo que cabría anticipar tratándose de un trabajo colaborativo, la traducción conserva

6 En esa misma ocasión, Emma Sentíes afirmaba que "Bassnett en esta compilación traducida no es solo Bassnett, es Bassnett con todas sus distintas vivencias y momentos entrando en resonancia con los traductores en cada uno de sus artículos" (2018, s/p). de manera homogénea y satisfactoria el estilo fresco, ingenioso y divertido de Susan Bassnett, la sutileza de su pensamiento y la intimidad de su escritura" (Calvillo, 2018, p. 313). Ojalá que, a pesar de la indispensable homogeneización para presentar la obra como una unidad, pueda verse la mano de cada uno de los participantes, pueda percibirse un dejo de su cosmovisión, de su historia personal y de su manera de relacionarse con la traducción como oficio y como disciplina.

Lo que fue originalmente una respuesta a la invitación a presentar proyectos relacionados con traducción que extendieron los editores de Bonilla Artigas en una presentación en la Sala Alfonso Reyes en El Colegio de México (Colmex), durante el Encuentro Internacional de Traductores Literarios que se lleva a cabo cada año en la unAm, el Colmex y el Instituto Francés de América Latina (IFAL), se concretó con el paso de varios años en la publicación de una traducción que originalmente no se pensó como un proyecto colectivo sino individual, a la manera tradicional. La editorial gestionó la participación del proyecto en el concurso del Programa de Apoyo a la Traducción (PROTRAD) al cual el FONCA contribuye con fondos para financiar la traducción de obras que un comité dictaminador considere importantes para la cultura de llegada. Parte de los requisitos de este programa es presentar el currículum del traductor que estará a cargo del trasvase de la obra, uno de los factores que el comité toma en cuenta para determinar la factibilidad del proyecto presentado. En el momento en que se publicaron los resultados del concurso, los cuales indicaban que nuestra propuesta había sido seleccionada para recibir los fondos, mis circunstancias académicas y personales habían cambiado enormemente en relación con las que tenía en el momento de proponer por primera vez el proyecto a la editorial, hacía más de dos años. Por esas y otras 
circunstancias no era posible que me hiciera cargo del proyecto en solitario y los editores de Bonilla Artigas tuvieron el valor de aceptar la propuesta de hacer la traducción por medio de un colectivo, que originalmente debía ser de menos de media docena de colegas, pero que se transformó hasta llegar a la decisión de asignar un capítulo a cada traductor.

Tras tomar la decisión tuvimos que seleccionar a aquellos colegas que por su talento, experiencia o preparación pudieran estar calificados para la tarea. Afortunadamente, casi en todos los casos que se extendió la invitación esta fue recibida con entusiasmo y con una respuesta afirmativa. Por cuestiones de derechos de autor, la editorial recibió el texto de parte de los agentes responsables de la obra original, con una marca de agua en cada página y la instrucción específica de no distribuir el texto ni compartirlo con terceros fuera del traductor y demás personas directamente involucradas. Por lo tanto, no me sentí en libertad de compartir la obra completa con los 39 colegas implicados ya en ese momento en el proyecto, a pesar de la total confianza que tenía en cada uno de ellos; este inconveniente se solucionó haciendo capturas de pantalla de cada página del libro y compartiendo con cada traductor solamente el capítulo al que se enfrentaría.

Gracias a las ideas que aportaban los miembros del colectivo se tomaron varias decisiones acerca de ciertos aspectos que debían estar definidos de antemano antes de que cada uno iniciara con su trabajo. Se establecieron ciertos lineamientos en cuestión de formato y estilo, entre ellos la sugerencia de la editorial de que buscáramos unificar un tanto la impronta personal que irremediablemente deja el traductor en su obra, es decir, teníamos que limitar necesariamente la polifonía producida por las distintas variantes del español de cada uno de los traductores involucrados. Esta decisión ha sido lamentada por algunos colegas que han comentado conmigo el asunto durante los meses posteriores a la publicación, ya que lo ven como la pérdida de un elemento que podría resultar sumamente interesante a nivel lingüístico y de la tan buscada visibilidad del traductor. Sin embargo, para una primera edición para la que no se había planteado de esta manera en las gestiones entre editoriales, creo que fue una decisión adecuada por parte de Bonilla Artigas, en especial porque contábamos con plazos muy estrictos dictaminados por las restricciones del PROTRAD, por lo que no era posible arrojarse a empezar una serie de discusiones con los editores del texto original para proponerles algo tan aparentemente osado. Nos encantaría (y no es plural mayestático, sino que hablo en nombre del Co-Laboratorio Traductor y sus diferentes integrantes) que en el futuro se presentara la posibilidad de tener una segunda edición, o de traducir otro texto de características similares (artículos o textos independientes) en donde las colegas colombianas hablaran en español colombiano, los mexicanos en nuestras distintas variantes regionales, y poder contar con la colaboración de colegas de otras latitudes con toda la riqueza de su propia lengua, de la "lengua de uno": "Reconocer la diferencia es un primer paso para comprender la otredad, con el fin de aprender a vivir con ello y adaptarse a comportamientos y conjuntos de valores distintos" (Bassnett, 2017, p. 209, trad. Karla Sánchez Domínguez). Al respecto, Mercedes Guhl señaló durante la presentación del libro, retomando una idea que figura en la tesis de doctorado de Sergio Bolaños, su colega y profesor en la Universidad Nacional de Colombia, citando a Frederick M. Rener, que los estudios de traducción vienen a ser "una especie de archipiélago constituido por muchas islas y ningún puente. Cualquier desarrollo de esta disciplina en una lengua dada queda circuns- 
crito a una de estas islas apartadas y sin comunicación con las demás." (Guhl, 2018, s. p.)

Una vez concluida la traducción, es decir, ya que cada uno de los traductores habían entregado puntualmente (la inmensa mayoría) sus respectivas traducciones, a las cuales habían dedicado largas horas de trabajo minucioso y revisado cuidadosamente (una vez más, la inmensa mayoría), tuvimos que darnos a la tarea de hacer una revisión lo más exhaustiva posible en un equipo de tres colegas. Distribuimos los 39 textos de manera que cada revisor trabajara con un bloque de 26 capítulos en el cual no se encontrara su propio texto, naturalmente. Es decir, cada capítulo fue revisado al menos por dos traductores en busca de inconsistencias, erratas, problemas sintácticos o semánticos, etc., cotejando siempre con los correspondientes capítulos del texto original. Tras las correcciones pertinentes (pocas en la mayoría de los casos, casi inexistentes en el caso de los traductores que combinaban los años de experiencia con una formación más profunda en la disciplina), se regresaba el texto a cada traductor para asegurarse de que estuviera de acuerdo con las sugerencias o correcciones realizadas, en un espíritu de respeto a su condición de autor de la traducción. ${ }^{7}$ Una vez que cada traductor aceptaba los cambios sugeridos a su texto, se "armó" todo el libro y se envió a una última revisión, esta vez por un colaborador externo, para hacer ya solo la corrección ortotipográfica final de la obra com-

7 En este punto estoy en deuda con el doctor Rafael Olea Franco, actual director del Centro de Estudios Lingüísticos y Literarios de El Colegio de México, quien fuera mi profesor tanto en la maestría en Traducción como en el doctorado en Literatura Hispánica. El doctor Olea Franco me hizo el favor de invitarme a colaborar en una de sus muy numerosas publicaciones colectivas y ahí, gracias a su ejemplo, aprendí la importancia de la comunicación con los colaboradores y del respeto que se les debe como autores de sus propios textos. pleta terminada para entregarla a la editorial. No puedo más que agradecer a cada uno de los colegas del colectivo que participaron en la traducción por su entusiasmo y buena voluntad hacia el proyecto, y muy particularmente al equipo reducido de tres colegas revisores que tuvieron la disposición y la dedicación infatigable para hacer las correcciones y sugerencias a los textos de los demás.

El amor por la traducción es algo que se transmite muchas veces de generación en generación y que se cultiva en pequeñas comunidades. Si volvemos nuestra mente a los inicios medievales de la conformación de los gremios, veremos cómo el oficio se aprende de la convivencia y el diálogo tanto con los pares como con los maestros que llevan años perfeccionándose en él y descubriendo nuevas maneras de acercarse a la materia prima para modelarla y obtener un producto a la vez útil y hermoso. Así lo aprendimos del testimonio de los grandes que vinieron antes que nosotros en De oficio traductor (Santoveña et al., 2010). Los trabajos presentes los desarrollamos, como decía Luis Vives siguiendo a Bernardo de Chartres, gigantium humeris insidentes - sobre hombros de gigantes-.

\section{La traducción colectiva como un proceso}

\subsection{El oficio en el taller: dos ejemplos de traducción literaria en colectivo a ambos lados del océano}

Tomás Segovia, profesor, poeta y traductor, autor del célebre trasvase de Hamlet al español, explica que aprender a traducir "es como para un carpintero aprender a incrustar maderas o para un deportista aprender a rematar de cabeza: hay que aprender. Hay una parte que es un don y hay otra parte que es poner 
mucha atención y ser humilde" (Santoveña et al., 2010, p.36). ${ }^{8}$ Se aprende, como todo oficio, poniendo manos a la obra y siguiendo el ejemplo de quienes tienen más experiencia en el mester. La traducción de textos para teatro es un ejemplo particularmente interesante del trabajo en colaboración y de cómo la humildad puede ser un requisito cuasi indispensable para el traductor teatral, no sólo porque muchas veces no recibe todavía crédito suficiente por su trabajo o porque su texto muchas veces no se cristaliza hasta alcanzar el estatus de texto publicado, sino por la necesaria interacción con otros agentes implicados en la puesta en escena:

[Cooperative translation] produces probably the best results. It involves the collaboration of at least two people on the making of the TL text-either an SL and a TL native speaker, or someone with knowledge of the SL who works together with the director and/ or actors who are to present the work. This method parallels the way in which theatre spectacle is created collaboratively, and the translator becomes someone who produces a basic scenario that is then worked on by the company (Bassnett, 1985, p. 91).

López Lapeña, siguiendo a Susan Bassnett, retoma el ideal de la traducción al alimón, contando con un equipo de dos traductores nativos: uno de la lengua fuente y otro de la lengua meta. En este fenómeno, al que denomina "traducción cooperativa" o "traducción colaborativa", participa más de un traductor "o un traductor que realiza su trabajo en estrecha colaboración con otros. En el caso del 'traductor a pie de escenario', el traductor rea-

8 Llama la atención que otro destacado escritor y traductor, Fabio Morábito, enfatice la importancia de esta misma cualidad: “¿Cuáles serían las características de un traductor literario? Bueno, humildad, justamente por el hecho de que sabemos que en el fondo es imposible traducir, que cada lengua es un universo intraducible" (Santoveña et al., 2010, p. 36). liza solo su trabajo y después lo somete a las decisiones de otros agentes" (López Lapeña, 2015, p. 96).

Los seminarios o talleres de traducción literaria son, entre los grupos que ejercen la traducción colectiva, los que mayor reflexión han propiciado, ya sea entre sus integrantes, ya sea al exterior de los mismos. Así lo explica Gabriel Linares: "Se ha traducido mucha literatura en México, pero siento que falta un poco de reflexión sobre la traducción y siento que los seminarios ayudan" (Santoveña et al., 2010, p. 165). Para ejemplificar la dinámica de trabajo que pueden desarrollar los colectivos quisiera presentar brevemente dos casos que han documentado el proceso que desarrollan para aproximarse a la traducción en colaboración, a saber, el Seminario Permanente de Traducción Literaria y el Taller de Traducción Literaria. El primero está integrado por profesores de la Facultad de Filosofía y Letras de la UNAM y el segundo, por un grupo más heterogéneo de alumnos, profesores, estudiantes de intercambio e incluso algunos participantes externos en la Universidad de La Laguna, en España.

Federico Patán, traductor de Shakespeare y profesor emérito de la UNAM, es miembro destacado del Seminario Permanente de Traducción Literaria. En entrevista plasmada en el volumen de testimonios colectivos De oficio, traductor. Panorama de la traducción literaria en México, Patán describe la dinámica de trabajo del grupo:

Cuando tenemos listo el borrador de la traducción lo intercambiamos. Se lo damos a tres colegas o a dos y nosotros a nuestra vez tomamos la traducción del colega y le hacemos todo el despedacerío que se puedan imaginar. Pero una de las reglas del Seminario es que el traductor tiene la última palabra. Si los tres que lo corregimos le decimos que esa palabra 
está mal traducida y él insiste en dejarla, pues que se quede si no lo hemos convencido, porque él es el responsable final del texto. (Santoveña et al., 2010, pp. 163-164)

Los distintos proyectos de traducción surgen a instancias de las propuestas de los propios integrantes, y una vez elegido el tema o el autor que dé unidad al proyecto, los traductores miembros del seminario seleccionan diferentes textos apropiados para el mismo. Ya durante la traducción en sí, para la toma de decisiones, Linares explica que "todo el tiempo vemos todo un proceso de negociación de significados. También hay un proceso por medio del cual cada quien defiende lo que cree que significa el texto o cómo el texto se debe expresar" (Santoveña et al., 2010, p. 165).

Acerca del Taller de Traducción Literaria, su creador y director, Andrés Sánchez Robayna explica cómo en un principio, hace más de dos décadas, enfrentaban la traducción de manera colectiva, pero "pronto descubrimos otras posibilidades engendradas o inspiradas por aquella, especialmente la traducción revisada, es decir, la traducción individual realizada por un miembro del grupo y más tarde revisada de manera colectiva por el resto de sus componentes" (Sánchez Robayna, 2007, p. 237). En el Boletín del Taller, así como en varios artículos al respecto (Sánchez Robayna, citado en $\mathrm{Cu}$ rell, 2018), Sánchez Robayna describe minuciosamente cómo llevaban a cabo el proceso de traducción per se de manera colectiva y las razones por las que se decantaron por el nuevo método que identifica como "traducción revisada". Al igual que en el caso del Seminario Permanente, en el Taller de la Universidad de la Laguna los integrantes presentan propuestas acerca de los textos que se van a traducir.

Estos dos colectivos, a ambos lados del Atlántico, son ejemplos icónicos, profusamente do- cumentados, y cuentan en su haber numerosas publicaciones, resultado de más de dos décadas de labor ininterrumpida. La comparación de Tomás Segovia sobre el traductor novel como aprendiz del taller puede aplicarse fácilmente a sus trabajos: "Es la diferencia entre una sabiduría y un conocimiento. Yo creo que la traducción no es conocimiento, es sabiduría. Idealmente, en un verdadero taller, entra un aprendiz y hay varios maestros trabajando" (Santoveña et al., 2010, p. 127). Las obras traducidas y publicadas por ambas agrupaciones de traductores son muestra de los frutos del esfuerzo comprometido, del respeto a los distintos puntos de vista de los colegas y de la humildad para recibir las opiniones de los demás miembros del grupo, muchas veces discordantes con la propuesta del traductor individual.

\subsection{Funciones de los agentes involucrados en el proceso}

La intención de este apartado es hacer una revisión de lo que muchos traductores ya desarrollamos en la práctica en cuanto a los proyectos colaborativos de traducción. Puede tratarse de "documentos pequeños relacionados entre sí. Digamos, facturas de una naviera o documentos relacionados con una demanda. En otras ocasiones pueden ser archivos muy extensos divididos o no en capítulos o secciones, por ejemplo, ensayos clínicos." (Sentíes, 2018). Como ya se apuntó, es fundamental conocer detalladamente el texto para saber cuáles de los integrantes del colectivo pueden desempeñar de manera más eficiente y con mejores resultados las diferentes tareas que suelen conformar este tipo de trabajos. Por los testimonios de los directores e integrantes de los grupos que se presentan en el apartado anterior, puede verse que dos virtudes primordiales para poder implementar una dinámica como esta son la humildad y el respeto: hu- 
mildad para reconocer las propias limitaciones y buscar ayuda en el miembro del equipo más capacitado para suplir esas carencias, y respeto para valorar las opiniones de todos los participantes y al mismo tiempo confiar en las decisiones de quien esté a cargo de cada proyecto específico.

La división de funciones dentro del "proyecto amplio de traducción" va mucho más allá que la traducción propiamente dicha. Estas funciones "van desde la atención al cliente [...], pasa por la cotización, la asignación del proyecto a los traductores, editores, diseñadores, terminólogos, revisores según corresponda, hasta los temas de cobranza, facturación y pago de impuestos" (Sentíes, 2018, s. p.). Al tratarse de una realidad en continuo movimiento, quizá no resulte muy productivo asignar papeles o responsabilidades de manera fija e inmutable. Los diferentes miembros de una comunidad traen consigo capacidades, experiencias y saberes distintos acumulados en la particular historia de vida de cada uno de ellos. Por lo tanto, es muy probable que quien pueda desempeñarse como terminólogo en un proyecto, por conocer mejor la jerga particular de una disciplina, necesaria para crear o revisar adecuadamente un glosario, quizá no pueda cumplir de la mejor manera esa función al tratarse de un proyecto nuevo de una materia distinta. Ese agente podrá tal vez aportar más al proceso al incorporarse en una función distinta, posiblemente la revisión ortotipográfica o la traducción perse.

En numerosos comentarios entre colegas, algunos plasmados en blogs sobre traducción, puede constatarse cómo una queja recurrente entre los egresados de programas universitarios de traducción es la falta de preparación para la realidad laboral. Si se toma en cuenta que la sistematización en los procesos de traducción colaborativa y la consecuente reflexión al respecto no han estado sobre la mesa durante mucho tiempo, probablemente sea uno de los grandes ausentes en los planes de estudio de las distintas instituciones educativas. Es de todos sabido que en traducción no hay recetas y que, al igual que en las ciencias médicas no hay enfermedades sino enfermos, en nuestro oficio cada texto requiere de un acercamiento particular determinado por sus características. Si se cuenta con la variable agregada de las distintas personalidades, capacidades y conocimientos de los diferentes agentes de una traducción colectiva, ese acercamiento es aún más individual. Sin embargo, lo que sí es verdad es que la dinámica de la traducción colectiva es un fenómeno presente en el mercado y que es deber de los docentes preparar a los alumnos para que puedan llegar mejor preparados a sus futuros trabajos en colaboración.

\section{Formación de traductores y percepción gremial de la traducción colectiva.}

Es un hecho de todos conocido que la lengua es como un organismo vivo en perpetua evolución. Sabemos también que la nuestra es una disciplina relativamente joven; aunque la traducción existe desde que existen las lenguas y desde hace siglos los traductores han reflexionado sobre su oficio (pensemos en Cicerón, en San Jerónimo y Lutero con sus traducciones de textos bíblicos, en Pushkin criticando la traducción que Chateaubriand hizo de Milton o en Nabokov explicando las razones por las que consideraba su traducción de Pushkin como uno de sus mayores logros), estamos conscientes de que la sistematización de dichas reflexiones y la conformación de la disciplina todavía tiene un largo camino por recorrer, y es muy emocionante formar parte de los peregrinos que recorren ese camino como los que fueron una vez en compañía de Chaucer en el 
camino a Canterbury. Por lo tanto, es difícil, si no es que imposible, pensar que puede haber una única propuesta docente, una sola manera adecuada de formar a los futuros traductores. Lo vemos en la diversidad de los programas universitarios de licenciatura, maestría y doctorado, así como en los distintos cursos y diplomados que se ofrecen en todo el mundo. Sin embargo, enfrentamos un reto incontrovertible: los docentes tenemos la obligación de formar a los traductores de las nuevas generaciones para que sean unos agentes pensantes y adaptables, preparados para enfrentar los cambios que la disciplina y la lengua nos presentan y que los adelantos tecnológicos nos obligan a adoptar de manera vertiginosa. Gertrudis Payàs, docente de la Universidad de Temuco, en Chile, a quien tuvimos el placer de tener como profesora en la maestría del Colmex, comenta acerca de las habilidades que identifica como necesarias para enfrentar la traducción en el mundo real, por ejemplo, en sus trabajos para Naciones Unidas: "[habilidades] detectivescas, de búsqueda de los orígenes de los textos, luego mecánicas, de traslado de esos fragmentos que ya están traducidos en otros textos, y finalmente de pasarle la plancha a todo eso para que quede uniformado" (Santoveña et al., 2010, p. 139).

Consciente de la importancia que tiene preparar a los estudiantes para que puedan enfrentar ese mundo real y no solo las situaciones hipotéticas que se enseñan dentro del salón de clases, Tomás Serrano, quien ha formado traductores durante varias décadas en la Universidad Intercontinental primero, y luego en el Colmex y la UNAM, señala que en relación a los alumnos que busca formar "me gustaría que pudiera ajustarse a las necesidades de una empresa editorial, que no son solamente de traducción, sino también de cotejo, de crítica, de dictámenes para recomendar a un cierto autor: todo el trabajo de la empresa editorial"
(Santoveña et al., 2010, p. 139). En un mercado laboral siempre cambiante, en el cual las habilidades del traductor no son solamente la de traducir, sino muchas otras, como se vio en las declaraciones anteriores y en la propuesta de trabajo presentada en el apartado 3, podemos darnos cuenta de que incentivar la flexibilidad y la resiliencia en los estudiantes es indispensable. Estas cualidades son de la mayor importancia cuando se desarrolla la labor en equipos de trabajo, tanto al interior del aula como en la vida profesional: "Within a relational paradigm, the translator is no longer a fixed intermediary between traditional binaries of source text/culture and target text/culture, he or she is now an active node in an evolving and dynamic web" (Cordingley y Manning, 2018, p. 4).

E1 traductor resulta entonces un elemento interrelacional, un integrante de esa red de expertos con los que tiene que vincularse para desarrollar su trabajo de la mejor manera posible. Por lo tanto, el desarrollo de habilidades interpersonales, si bien damos por hecho que debe enseñarse en otra etapa de la formación de los alumnos, no debe descuidarse en la formación profesional. Es nuestro deber hacer conscientes a los estudiantes de la importancia de mantener una interacción saludable con los demás agentes involucrados en el desempeño del oficio, sean correctores, editores, promotores de lectura, clientes, y naturalmente con otros traductores. Así lo declara el doctor Gabriel Linares al hacer referencia al trabajo colaborativo desarrollado en el Seminario Permanente, pero su aseveración bien puede extrapolarse a todos los campos de trabajo colaborativo relacionadas con la traducción: "Se necesita flexibilidad para que los miembros entiendan a los otros miembros, pero también a la vez se necesita rigor; si no hay afinidad, si no hay rigor, disciplina, es difícil que funcione" (Santoveña et al., 2010, pp. 165-166). 
Si comprendemos que solo somos un nodo de esa red en movimiento a la que se refieren Cordingley y Manning; si logramos hacer conciencia, primero en nosotros y después en los estudiantes, de que como traductores somos apenas un agente (aunque fundamental en el proceso) que requiere ineludiblemente de la participación de los demás agentes involucrados en el proceso traductor, que no puede desarrollar su trabajo con la misma calidad y eficiencia sin la colaboración de colegas traductores, de revisores, de gestores de proyecto, de expertos en el área de especialidad del tema que traducimos, podremos tener una actitud más humilde frente a la inmensidad de nuestra tarea y frente a la importancia del trabajo colaborativo y del aprendizaje continuo. Retomando la figura del taller medieval, Tomás Segovia afirma de manera contundente que "la formación de traductores es como un oficio. Idealmente, sería un aprendizaje como el de un aprendiz: estar cerca del maestro que tiene más experiencia, robándole las técnicas y las ideas, imitándolo" (Santoveña et al., 2010, p. 127). Me atrevería a extrapolar la comparación que hace el poeta traductor de Hamlet no solo a la formación de traductores sino a la formación de formadores, un campo muy abandonado en todas las disciplinas profesionales y la nuestra no es la excepción. También los formadores aprendemos de otros formadores, también los imitamos y les robamos las ideas y las técnicas, a veces de manera consciente y explícita, otras, no tanto.

Nair Anaya, gran formadora de traductores en El Colegio de México, comparte su experiencia en los primeros años de su participación en el Seminario Permanente de la UnAM: "Nos revisamos unos a otros y al final hacemos una revisión general. Eso es muy enriquecedor [...] Hay gente con mucha más experiencia que otra. Cuando yo empezaba me sirvió muchísimo que me desbarataran entre todos y aprendí mucho" (Santoveña et al., 2010, p. 163). Se ve que aprendió bien el método durante esos años y que consideró que valía la pena llevarlo a sus propias aulas del Colegio al formar a muchas generaciones en su clase de Prácticas de traducción. El sistema funcionó, evidentemente, al formar a muchos estudiantes que ahora ejercen la traducción de manera profesional, pero también al dar ejemplo para que pudiéramos imitarlo quienes nos hemos dedicado años después a la docencia. Confiamos en que la práctica hace al maestro y en que esas "imitaciones" de los aprendices sean cada vez más cercanas al modelo propuesto.

Por otra parte, Monique Legros, fundadora del PFT, Programa de Formación de Traductores, precursor de la maestría en traducción en El Colegio de México, el cual dirigió durante alrededor de veinte años, apunta al respecto de la práctica colaborativa entre los estudiantes del programa:

Entre 1974 y 1995 el PFT acogió alumnos extranjeros de veintinueve nacionalidades. El que hablaran español además de su propio idioma permitió, en ciertos casos, la constitución de equipos de dos alumnos en las prácticas de traducción: uno que dominaba el español y uno que dominaba su lengua —inglés, francés, alemán, italiano—. Los resultados eran buenos y no vacilé en recomendarlo no solamente en la clase sino en la práctica profesional. Según los que lo practicaban, ahorraban la mitad del tiempo y adquirían una seguridad mayor, una confianza mayor, para cada uno, en la calidad de la traducción así efectuada. (Santoveña et al., 2010, p. 217)

Sabemos que normalmente no es posible contar con estudiantes extranjeros con los cuales organizar mancuernas de este tipo. Sin embargo, es esencial no solo presentarles a los alumnos las posibilidades que presenta la revisión mutua, sino demostrarles los beneficios de practicarla, llevando a cabo prácticas de traducción en las que no se incluya la revisión 
inter pares para después incluirla en el proceso y dejar que hagan un análisis comparativo de la calidad de los resultados y de la mayor rapidez con la que pueden desarrollar dicho proceso de principio a fin. Lo mismo ocurre con el hecho de generar conciencia de la importancia de especializarse en cierta temática o tipo de textos, o contar, como alternativa, con la presencia de un experto en el tema al cual pueda acudirse para las etapas de revisión o gestión terminológica. Por lo general el cliente o algún miembro de su equipo cuenta con conocimientos especializados sobre el tema, pero no siempre tiene la capacidad o la disposición para llevar a cabo la traducción; el especialista de la compañía que encomienda la traducción "may not be able or willing to translate your document into French, but you may be able to enlist her as a subject matter expert (SME) to review the translation provided by a qualified professional translator" (Russi y Schneider, 2016, p. 12). En el aula esto puede mostrarse de manera relativamente fácil al propiciar que los alumnos lleven a cabo simulaciones periódicas de encargos de traducción en las que cada uno de ellos haga el doble papel de cliente que hace el encargo y de experto que puede asesorar al resto del grupo en cuestiones terminológicas y que al final lleva a cabo una revisión exhaustiva de la calidad de las traducciones. Si el "cliente/experto" es quien propone la traducción de un texto dentro de su área de especialidad, generalmente tiene de antemano una idea más clara de los vericuetos específicos de ese tipo de textos y de los usos apropiados de los términos, por lo que puede ofrecer una retroalimentación aguda y detallada. En el último semestre, durante las prácticas de traducción en la maestría en el Colmex, tuvimos oportunidad de experimentar este tipo de dinámicas con la profesora Nair Anaya. Varios de sus discípulos que nos dedicamos actualmente a la enseñanza seguimos su ejemplo al implementar esta estrategia en clase con consecuencias positivas, visibles en las traducciones resultantes y en la percepción de los estudiantes.

El diseño de los planes y programas de estudio forzosamente tiene que estar vinculado con la realidad cambiante, adaptarse a ella y por lo tanto reformularse de manera periódica. Recientemente he compartido impresiones con alumnos y colegas, así como con otros informantes, acerca de la percepción que tenemos en el gremio, en el contexto de la traducción literaria y especializada en México y en algunos otros lugares de América Latina y España. Tuve oportunidad de complementar esta información con una encuesta en línea cuyos resultados refuerzan la percepción de muchos de nosotros al respecto: la traducción colaborativa está cada vez más presente en el panorama laboral al que nos enfrentamos día con día (más del $70 \%$ han participado en proyectos que la involucran); la reflexión acerca de esta práctica es indispensable para que las condiciones en que se lleva a cabo sean más favorables para los distintos agentes involucrados en el proceso; las distintas funciones que cumplen estos agentes deben analizarse, sistematizarse, redefinirse, para tener conciencia de las distintas posibilidades que se presentan al desarrollar el trabajo de manera colectiva.

En las conversaciones y en los incipientes resultados de la encuesta se refleja la importancia creciente (y en consecuencia el porcentaje de presupuesto asignado) que se concede a la etapa de corrección del producto final, solo por debajo de la traducción perse, pero muy por encima de la gestión del proyecto y la búsqueda terminológica. Este último aspecto lo explican por la mayor disponibilidad de herramientas de búsqueda y de creación de glosarios compartidos en los que todos los integrantes del proyecto contribuyen en tiempo real. La temática de los textos que consideran apropiadas 
para el proceso colaborativo de traducción es cada vez más amplia, incluyendo la traducción especializada de textos científicos, técnicos y de la industria farmacéutica, textos legales y financieros, de humanidades y literarios, e incluso se menciona la novela gráfica. También destaca entre las impresiones compartidas la importancia de distribuir adecuadamente las labores de los distintos agentes implicados y de delimitar con claridad las funciones que desarrolle el gestor del proyecto, entre las que se incluyen la elaboración del manual de estilo y el glosario, la edición del producto terminado, el manejo de los documentos originales y el cuidado en la edición de los mismos previo a la distribución de las tareas o los fragmentos para evitar fugas de información delicada por los acuerdos de confidencialidad, la búsqueda de patrocinios para nuevos proyectos y la resolución de imprevistos.

\section{Conclusiones}

Las oportunidades de participar en proyectos colectivos de traducción son cada vez más frecuentes; los textos que se proponen para este tipo de trabajo incluyen una variedad creciente de temáticas y tipologías textuales. Probablemente esto se deba, al menos en parte, al cuestionamiento actual sobre la figura del autor, pero con seguridad se alimenta de los avances tecnológicos que nos permiten realizar con mayor facilidad tareas que involucran una comunicación constante con los integrantes del proyecto. En su reflexión acerca de la naturaleza colaborativa de la traducción, Emma Sentíes apunta:

En el contexto de los argumentos en contra de la traducción descrita como una pérdida necesaria, Lefevere reivindica el papel de la traducción y explica, más o menos así: El fenómeno de la traducción es como el del arcoíris. La luz en este caso sería el texto original, el proceso de traducción es el prisma por el que pasa esta luz, y las traducciones que resultan, las traducciones como producto, son los colores en los que esa luz se refracta. La traducción no resta, suma. (2018, s. p.)

Los distintos saberes de los miembros de un colectivo de traducción, sus distintas habilidades, conocimientos y destrezas contribuyen al crecimiento de esa persona comunitaria que tiene que enfrentarse al texto. Cada elemento del equipo aporta sus conocimientos, sus experiencias de vida, su visión del mundo que se complementa con la de los demás y enriquece el resultado final. Es fundamental reconocer las fortalezas de cada uno de los integrantes, pero también poder identificar cuál es el talón de Aquiles de aquellos que conforman esa comunidad. Fabienne Bradu declara sin ambages su convicción en que el traductor no tiene por qué ser ese "animal de soledades" que nombraba Rosario Castellanos:

Yo quisiera romper la soledad del traductor porque llega un momento, después de revisar tantas veces una página, una escena, un poema, que uno ya no ve; uno necesita otros ojos, otros oídos y, entre más finos sean esos ojos y esos oídos, mejor. Uno tiene que tomar una distancia y tener una opinión, que a fin de cuentas es el mismo mecanismo que cuando uno termina un libro y se lo da a leer a alguien. Siempre hay una aportación, un enriquecimiento del otro que se necesita. No creo mucho en la soledad del traductor. Creo más en las mancuernas. (Santoveña et al., 2010, p. 220)

El correcto funcionamiento de la maquinaria de cualquier tipo de colectivo es responsabilidad de todos los que se constituyen en un engrane al integrarse a este. En el caso de la traducción colectiva, la conciencia de esta responsabilidad compartida propicia que se busque identificar quién es el agente ideal para cumplir con las distintas tareas implicadas en un proceso colaborativo, lo cual trae como consecuencia un producto más pulido, un pro- 
ceso más eficiente y mejores condiciones de trabajo para todos.

\section{Referencias}

Bassnett, S. (1985). Ways through the labyrinth: Strategies and methods of translating theatre texts. En T. Hermans (ed.), The manipulation of literature: Studies in literary translation, Londres: Croom Helm.

Bassnett, S. (2011). Reflections on translation. Bristol: Multilingual Matters.

Bassnett, S. (2017). Reflexiones sobre traducción. (Varios, trad.; M. Celis, coord. trad.). Ciudad de México: Bonilla Artigas Editores.

Calvillo, J. C. (2018). Reseña de Reflexiones sobre traducción. (Varios, trad.; M. Celis, coord. trad.). Trans, Revista de Traductología, (22), 311-313. Recuperado de http:// www.revistas.uma.es/index.php/trans/ article/view/5107/5118

Cordingley, A. y Manning, C. F. (eds.) (2018). Collaborative translation: From the Renaissance to the digital age, Londres: Bloomsbury.

Curell, C. (2018). Diálogo con Andrés Sánchez Robayna acerca del taller de traducción literaria de la Universidad de la Laguna. Tropelías. Revista de Teoría de la Literatura y Literatura Comparada, (29), 241249. Zaragoza: Universidad de Zaragoza. DOI: https://doi.org/10.26754/ojs_tropelias/tropelias. 2018292563

Eco, U. (1993). Traduction et langue parfaite. En Dixièmes assises de la traduction littéraire. Arles: ATLAS. Recuperado de https:// www.atlas-citl.org/10-e-assises-de-la-traduction-litteraire-arles-1993/

Foz, C. (2000). El traductor, la Iglesia y el rey. La traducción en España en los siglos XII y XIII. Barcelona: Gedisa.

González Velázquez, C. A. (2018). Presentación de Reflexiones sobre traducción (texto inédito). Recuperado de http://co-labora toriotraductor.blogspot.mx.
Guhl, M. (2018). Presentación de Reflexiones sobre traducción, de Susan Bassnett (texto inédito). Recuperado de http://co-laboratoriotraductor.blogspot.mx.

Lindner, A. (2006). Lineas conectadas: nueva poesía de los Estados Unidos. (Varios, trad.). Louisville, KY: Sarabande.

López Lapeña, A. (2015). Traducir un arte vivo. Bases para un modelo de análisis de la traducción teatral [tesis doctoral]. Universidad de Granada. Recuperado de https://hera. ugr.es/tesisugr/24798745.pdf

López Mills, T. (2011). Traslaciones. Poetas traductores 1939-1959. Ciudad de México: Fondo de Cultura Económica.

Montes de Oca, A. y Montes de Oca Vega, A. L. (1974). El surco y la brasa. Ciudad de México: Fondo de Cultura Económica.

O'Brien, S. (2011). Collaborative translation. En Y. Gambier y Luc van Doorslaer (Eds.), Handbook of Translation Studies (vol. 2). Amsterdam: John Benjamins.

Paz, O. (1971). Traducción: literatura y literalidad. Barcelona: Tusquets.

Russi, D. y Schneider, R. (2016). A guide to translation project management, UCAR/COMET. Recuperado de https://courses.comet. ucar.edu/pluginfile.php/27060/mod_resource/content/12/GuideToTranslationManagement_V1a_02102017_final.pdf

Sacks, O. (2008). El hombre que confundió a su mujer con un sombrero (J. M. Álvarez Flórez, trad.). Barcelona: Anagrama.

Sánchez Robayna, A. (2007). Traducir, esa práctica. En J. Doce (Ed.), Poesía en traducción. Madrid: Círculo de Bellas Artes.

Sánchez Robayna, A. (2017). Renga en la tradición del texto colectivo. En E. Ramos-Izquierdo y P. H. Giraud (Eds.), Lecturas/Lectures de Renga. París: Colloquia.

Santoveña, M., Orensanz, L., Leal Nodal, M. A. y Gordillo, J. C. (2010). De oficio, traductor. Panorama de la traducción literaria en 
México. Ciudad de México: Bonilla Arti- Sentíes Miranda, E. (2018). Presentación de gas Editores/CONACULTA/FONCA. Reflexiones sobre traducción (texto inédito). Seféris, Y. (2008). Novela (Mythistórima)(S. Ancira y F. Segovia, trads.). Ciudad de MéRecuperado de http://co-laboratoriotraxico: Ediciones Sin Nombre/Vaso Roto. ductor.blogspot.mx.

Cómo citar este artículo: Celis-Mendoza, M. (2019). La traducción colectiva como proceso o producto. Reflexiones sobre el trabajo en colaboración a partir de casos de estudio concretos. Mutatis Mutandis. Revista Latinoamericana de Traducción, 12(2), 540-558. DoI: 10.17533/udea.mut. v12n2a10 